\section{The Disconnection between Mental Health and Substance Use Treatment: The Need for the Integrated Care/Treatment Model}

\author{
Douglas J Muller* \\ Department of Psychology, Hampton University, Virginia, USA
}

When I first entered the Substance Use Disorder (SUD) field in 1980 as a Drug/Alcohol Abuse Control Officer in the Air Force, I did not know at the time that I would be part of one of the most effective alcohol treatment programs. Being new to the field I did not realize that the training and experiences at that time would be the "gold standard" for the rest of my career in substance use counseling. My training was unique because I attended two different programs that between them addressed both the drinking behavior typically associated with substance use but as well as the emotional factors that impact the individual and his/her family. Despite the intensive training, I felt overwhelmed as I walked into my first group counseling session. To my relief I was partnered with a co-facilitator who was the psychologist in charge the organization's mental health program. It was not till a year later the "light went on" when I realized that together we were able to utilize each other's strengths, perspectives, and insights to cover both the alcohol/drug behavioral issues as well as their mental health concerns of our clients. Each session, we collaborated and continued after each session to discuss the intersections between my observations of our clients' behavior and his observations of their mental processing. At one point, the group did not feel that the psychologist provided any assistance to their recovery program since he focused on mental health and the clients perceived they "only" had a drinking problem. To demonstrate why an integrated approach was necessary, my co-facilitator asked the group to complete the Minnesota Multiphasic Personality Inventory (MMPI). After obtaining the results, the psychologist due to observing the group members' behavior over several sessions was able to identify which set of MMPI results belonged to each client. This collaboration between mental health and substance use professionals demonstrated to the group members, including myself as well as that their substance

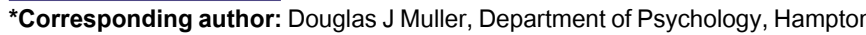
University, Virginia, USA, Tel: +1 7577272314; E-mail: douglas.muller@hamptonu.edu

Citation: Muller DJ (2016) The Disconnection between Mental Health and Substance Use Treatment: The Need for the Integrated Care/Treatment Model. J Alcohol Drug Depend Subst Abus 2: 003.

Received: February 29, 2016; Accepted: March 01, 2016; Published: May 13, 2016 use behavior was directly connected to their mental/emotional processes. I learned very early in my career that substance use providers need to be aware of the possibility of additional mental health issues. This awareness can be developed through an increased partnership between substance use and mental health professions. If this partnership has not been developed then there is a disconnection. For example, having done research on adult Attention Deficit Disorder (ADD), which is the inattentive side of Attention Deficit Hyperactivity Disorder (ADHD), it reinforced my perspective that by focusing solely on the substance use behavior that additional symptoms or mental health disorders may be missed thus sabotaging both treatments. To emphasis this point, Kevin Patrick in his book "No Way to be Treated: Managing Addiction by Treating ADHD" states, "Treatment has it wrong; backward is a better choice of words. It seeks to treat the addiction and let the presence of ADHD go unrecognized. Once treatment programs learn to treat the ADHD along with the addiction, they will be astonished at the result" [1]. Though the book's focus is on ADHD, almost any mental health disorder can be substituted in its place. I continue to take this approach to gain insight into the multilayer of emotions and behaviors of substance users and that by not including mental health issues in addition to substance use, treatment will not be as effective. This example of the Integrated Care/Treatment Model is not a new concept but there has been an on-going disconnect between chemical addiction and mental health professionals.

Researching material for this editorial article, I found a handout from a workshop I attended provided by Hazelden in 1992 and titled "Dual Disorders in the Chemically Dependent Client" that demonstrated the value of chemical dependency and mental health professionals working together to assist addictive clients with co-occurring disorders. One of the primary issues they addressed was, "Why mental health staff don't like to refer to chemical dependency counselors". The following concerns were addressed: 1 . they simplify everything down to addiction; 2 . they influence the client against mental health treatment; 3 . they encourage the client to stop using medications; 4. I don't get the client back; 5. their treatment focus is too simplistic. The second concern they addressed was, "Why chemical dependency counselors don't like to refer to mental health staff". The following concerns were addressed: 1 . they simplify everything down to the mental illness problem; 2 . they influence the client against addiction treatment; 3 . they use diagnostic criteria that doesn't take alcohol/drug use into consideration; 4. I don't get the client back; 5. their treatment focus overlooks addiction [2]. If any of these statements sound familiar, then possibly the addiction field has not progressed or evolved due to continuing non-acceptance of individuals outside of our specific professions. These disconnections might suggest that providers and therapists are not seeking an integrated approach denying their clients the necessary treatment for co-occurring disorders by not referring to or collaborating with other practitioners.

I suggest that practitioners broaden the focus of their pedagogy in order to strengthen the Substance Use/Addiction field therefore recommend the following areas/issues that need to be addressed: 
1. Co-occurring disorders not being considered

2. The relationship and interaction between substance use and mental health disorders

3. Integrate treatments for substance use and mental health

4. Co-facilitation/consultation between substance use and mental health providers

5. Self-medicating issues that are not taken into account during treatment

6. Misdiagnoses due to overlapping substance use and mental health symptoms

7. Training to recognize mental health symptoms by substance use providers

I believe this journal can provide the opportunity to address these issues through our awareness of the need for continuing research and best practice methods when treating those suffering from substance use disorders or addictions. We can also provide knowledge of and access to the appropriate training to develop our skills and available resources that reinforce the Integrate Care/Treatment Model. This model also takes into account the need and provisions for the co-dependentfamily members. All too often the impact of the disorder/co-occurring disorders on family members are not treated or taken into consideration. As I learned over 35 years ago, successful treatment of the client means we must address multilayered issues and that no single technique works for every client or provider. Gaining knowledge, training, and experiences outside of a provider's comfort zone, creates the opportunity to expand their theories and perceptions of addiction disorders. As scientific evidence produces greater insight of how physical and psychological dynamics interact, the substance use/addiction field needs to continue to develop and grow to utilize this knowledge in its treatment practices. I look forward to learning from other providers their unique insights on substance use treatment; and thank them for their dedication to improving the lives of those with addictions.

\section{References}

1. Patrick K (2010) No way to be treated. Tate Publishing \& Enterprises, Mustang, OK, USA.

2. Hazelden (1992) Dual disorders in the chemically dependent client. Burgin JE (Trainer). Community prevention and professional education. Symposium conducted at the Virginia Project for Addiction Certification Training, Norfolk, VA. 